\title{
Improved rubidium atomic beam clock based on lamp-pumping and fluorescence-detection scheme
}

Y. H. Wang

wangyanhui@pku.edu.cn

\section{J. Q. Huang}

Y. Gu

S. Q. Liu

T. Q. Dong

Z. H. Lu

\author{
Institute of Quantum Electronics, School of Electronics Engineering \& Computer Science, Peking \\ University, Beijing 100871, P. R. China \\ Institute of Quantum Electronics, School of Electronics Engineering \& Computer Science, Peking \\ University, Beijing 100871, P. R. China \\ Institute of Quantum Electronics, School of Electronics Engineering \& Computer Science, Peking \\ University, Beijing 100871, P. R. China \\ Institute of Quantum Electronics, School of Electronics Engineering \& Computer Science, Peking \\ University, Beijing 100871, P. R. China \\ Institute of Quantum Electronics, School of Electronics Engineering \& Computer Science, Peking \\ University, Beijing 100871, P. R. China \\ Max Planck Institute for the Science of Light, and University Erlangen-Nuremberg, 91058 Erlangen, \\ Germany
}

A compact, portable rubidium atomic beam clock based on lamp-pumping and fluorescence-detection scheme is proposed. The expected short-term frequency stability can be at least three orders of magnitude better than that of previous experimental results. The usages of lamp pumping, fluorescence detection and microwave slow-wave resonance structures make this design robust and compact. [DOI: $10.2971 /$ jeos.2011.11005]

Keywords: lamp-pumping, fluorescence-detection, atomic beam clock, frequency stability

\section{INTRODUCTION}

Techniques developed in recent years to produce and manipulate cold atoms or cold ions are expected to lead to rapid improvements in atomic clocks. The development of an atomic fountain clock based on laser-cooled atoms has created prospects for a considerably improved accuracy and stability of the cesium frequency standard. But most of them are running in scientific laboratories and require professional scientific researchers for day-to-day maintenance. On the other hand, there are still large markets for care-free beam clocks, especially small size, portable beam clocks, like Agilent 5071A. This robust cesium beam clock is widely used in time keeping, satellite navigation and deep space exploration.

In comparison with rubidium cell clock, the stability of cesium beam clock is better, mainly due to better signal to noise ratio $(\mathrm{S} / \mathrm{N})$ achievable in the beam sample. However, there are no special reasons why the same technical improvements used for the cesium beam clock could not be applied to the rubidium beam clock. For the development of portable beam clocks, a compromise must be found among the achievable accuracy and stability and the corresponding physical designs, power consumptions, and costs. The purpose of this paper is to present a scheme of rubidium atomic beam clock as an alternative choice for cesium beam clock. The new design will have similar performance levels as a cesium beam clock, but will be much more compact.
The initial idea and preliminary experimental results of an optically pumped rubidium beam clock was reported by Arditi et al. around thirty years ago [1]. Later, a detailed theoretical analysis of the operation of this optically pumped rubidium beam clock was developed [2]. From the analysis, it was concluded that the performance limitation of this kind of beam clock was dominated by the poor $\mathrm{S} / \mathrm{N}$ of the optical detection signal.

With the development of the diode lasers, a few groups started to work on the laser-pumped cesium and rubidium beam clocks [3]-[7]. The obvious advantages are the higher utilization rate of the atoms and the more compact design in comparison with the traditional magnetic state selection type beam clocks. Experimental results demonstrated the expected improvements in short-term stabilities. Unfortunately, due to the frequency drift of the diode lasers, the long-term performance level is not so great. This limits the further improvements and the marketing of the products. Up to now, as far as we know, there are still no commercial laser-pumped cesium or rubidium beam clocks available.

In this paper, we propose to use the rubidium lamp as pumping source but with a different pumping scheme. The specific pumping scheme is selected from four possible pumping schemes. The selected pumping scheme has the highest pumping efficiency. In addition, a fluorescence detection tech- 


\begin{tabular}{|c|c|c|c|c|c|c|}
\hline Sample & Number & $\begin{array}{c}\text { Pumping } \\
\text { light }\end{array}$ & $\begin{array}{c}\text { population } \\
\text { level }\end{array}$ & $\begin{array}{c}\text { Detecting } \\
\text { light }\end{array}$ & $\begin{array}{c}\text { Utilization } \\
\text { rate }\end{array}$ & $\begin{array}{c}\text { Signal to } \\
\text { noise ratio }\end{array}$ \\
\hline${ }^{87} \mathrm{Rb}$ & 1 & $\mathrm{a}($ or $\mathrm{A})$ & $\mathrm{F}=1$ & $\mathrm{~b}$ & $1 / 3 \times 0.278$ & $1 / 3 \times 0.278 \times 0.278=0.026$ \\
\cline { 2 - 7 } & $\mathbf{2}$ & $\mathbf{b}$ & $\mathrm{F}=\mathbf{2}$ & $\mathbf{b}$ & $\mathbf{1 / 5} \times \mathbf{0 . 2 7 8}$ & $\mathbf{1 / 5} \times \mathbf{0 . 2 7 8 = 0 . 0 5 6}$ \\
\hline${ }^{85} \mathrm{Rb}$ & 3 & $\mathrm{~A}(\mathrm{or} \mathrm{a})$ & $\mathrm{F}=2$ & $\mathrm{~B}$ & $1 / 5 \times 0.722$ & $1 / 5 \times 0.722 \times 0.722 \times(3.036 / 6834)=0.046$ \\
\cline { 2 - 7 } & 4 & $\mathrm{~B}$ & $\mathrm{~F}=3$ & $\mathrm{~B}$ & $1 / 7 \times 0.722$ & $1 / 7 \times 0.722 \times(3.036 / 6834)=0.046$ \\
\hline
\end{tabular}

TABLE 1 Different combinations of pumping light and sample atoms for rubidium beam clock.

nique is selected to suppress the background noise. The detected signal level is further increased by the use of more photo-detectors for photo detection. In this paper, the operation of the clock system is explained. The short-term frequency stability of the proposed rubidium beam clock is calculated, demonstrating an at least three orders of magnitude better performance level than previous experimental results.

\section{LAMP PUMPING OF A RUBIDIUM BEAM CLOCK}

Rubidium has ${ }^{85} \mathrm{Rb}$ and ${ }^{87} \mathrm{Rb}$, two isotopes with the abundance of $72.2 \%$ and $27.8 \%$, respectively. The $\mathrm{D}_{2}$ hyperfine transition lines of ${ }^{85} \mathrm{Rb}$ are designated as $\mathrm{A}$ and $\mathrm{B}$ lines, and the $\mathrm{D}_{2}$ hyperfine transition lines of ${ }^{87} \mathrm{Rb}$ are designated as $\mathrm{a}$ and $\mathrm{b}$ lines as shown in Figure 1. Components A and a are nearly coincident while components B and b are completely separated. In the experiment of Arditi et al. [1], ${ }^{87} \mathrm{Rb}$ atoms are illuminated with the resonant light from a lamp containing ${ }^{85} \mathrm{Rb}$ atoms. Component $\mathrm{A}$ of the lamp light will optically pump the ${ }^{87} \mathrm{Rb}$ atoms into $\mathrm{F}=1$ ground state.

In addition to the above pumping scheme, there are other possible pumping schemes. The rubidium lamps can be filled with ${ }^{87} \mathrm{Rb}$ atoms, and the working atoms can also be ${ }^{85} \mathrm{Rb}$ atoms. Therefore, there are four possible combinations as shown in Table 1. In Table 1, the first method is exactly the method of Arditi et al. [1]. Considering the hyperfine splitting of the ground state energy levels and the natural abundance of $\mathrm{Rb}$ atoms, the utilization rate of the ${ }^{87} \mathrm{Rb}$ working atoms is $1 / 3 \times 0.278$. For a non-isotope-enriched rubidium oven, the ${ }^{85} \mathrm{Rb}$ in the beam will absorb the photons from the pumping lamp. As a consequence, most pumping light is "wasted". The final S/N is proportional to the square of the natural abundance of $\mathrm{Rb}$ atoms. For the first method, it is $1 / 3 \times 0.278 \times 0.278=0.026$. The second method also uses ${ }^{87} \mathrm{Rb}$ as sample atoms, and utilizes ${ }^{87} \mathrm{Rb}$ lamp pumping $\left({ }^{85} \mathrm{Rb}\right.$ filter). This method has the highest rubidium atoms utilization rate. This change should improve the $\mathrm{S} / \mathrm{N}$ by a factor of two. The pumping efficiency can be further improved by using a bigger light bulb with $32 \mathrm{~mm}$ diameter filled with pure ${ }^{87} \mathrm{Rb}$ and 2 Torr $\mathrm{Kr}$ buffer gas. The emitted light power is proportional to the volume of the bulb. The noise can be kept in a similar level after careful designing of the light bulb electronics circuit and adjusting the working parameters. Therefore, using a larger light bulb can improve the $\mathrm{S} / \mathrm{N}$ by a factor of $(32 / 25)^{3}=2$ in comparison with a $25 \mathrm{~mm}$ diameter light bulb. In our lab, we have already finished building the larger light bulb. The measured power and noise are in accordance with our estimation.
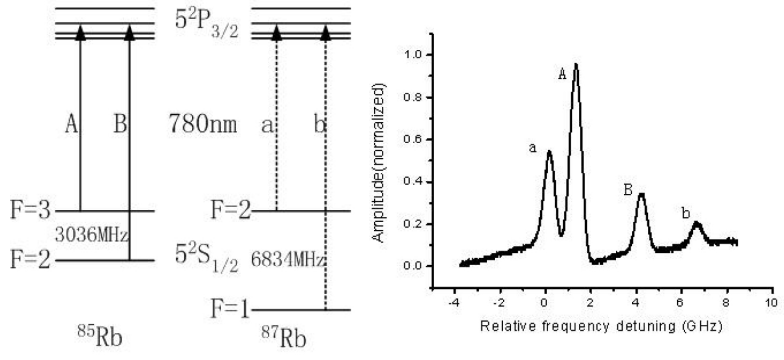

FIG. 1 Hyperfine structure and $D_{2}$ lines of ${ }^{85} \mathrm{Rb}$ and ${ }^{87} \mathrm{Rb}$.

\section{FLUORESCENCE DETECTION TECHNIQUE}

For the second method, transition line $b$ is used for the fluorescence detection instead of transmission detection. When the microwave frequency is tuned away from the resonance of the $\mathrm{F}=2, \mathrm{~m}=0$ to $\mathrm{F}=1, \mathrm{~m}=0$ transition $(0-0$ clock transition), the signal output is in a low level, which constitutes the background noise. The signal output is high if the microwave frequency is resonant with the $0-0$ clock transition. For transmission detection, the absorbed power is $P_{a b s}=\sigma_{a b s} \Delta l N P_{\text {in }}$, where $\sigma_{a b s}=1.938 \times 10^{-9} \mathrm{~cm}^{2}$ is the absorption cross-section for $\mathrm{D}_{2}$ transition, $\Delta l=0.3 \mathrm{~cm}$ is the interaction path-length, $N=10^{6} \mathrm{~cm}^{-3}$ is the atomic number density in the interaction region, $P_{\text {in }}=10^{-3} \mathrm{mw}$ is the rubidium lamp's power at $780 \mathrm{~nm}$. The responsivity of the photo detector S5106 (Hamamatsu) is $R=0.57 \mathrm{~A} / \mathrm{W}$ at $780 \mathrm{~nm}$. Assuming the noise is dominated by the shot noise, then the calculated $\mathrm{S} / \mathrm{N}$ is

$$
\frac{R P_{a b s}}{\sqrt{2 q R P_{i n} \Delta v}}=776,
$$

where $\mathrm{q}$ is the electron charge, $\Delta v$ is the detection bandwidth in Hertz (in calculation, $\Delta v=1 \mathrm{~Hz}$ ). For fluorescence detection, the detected fluorescence power is $P_{f l}=\delta \Gamma P_{a b s}$, where $\delta=0.005$ is the fluorescence collection efficiency, $\Gamma=\frac{T}{2 \tau}=1080$ is the atomic fluorescence cycle during its transit through the interaction region. Here $T=5.6 \times 10^{-5} \mathrm{~s}$ is the atomic transit time; $\tau=2.6 \times 10^{-8} \mathrm{~s}$ is the excited state lifetime. The calculated fluorescence collection $\mathrm{S} / \mathrm{N}$ is

$$
\frac{R P_{f l}}{\sqrt{2 q R P_{f l} \Delta v}}=74780 .
$$

In comparison with transmission detection, the improvement of $\mathrm{S} / \mathrm{N}$ is approximately 96 . The improvement is also in accordance with silver's experimental results [8].

Since the time constant of the servo-loop can be in the range of second, multiple photo-detectors can be used to detect the flu- 


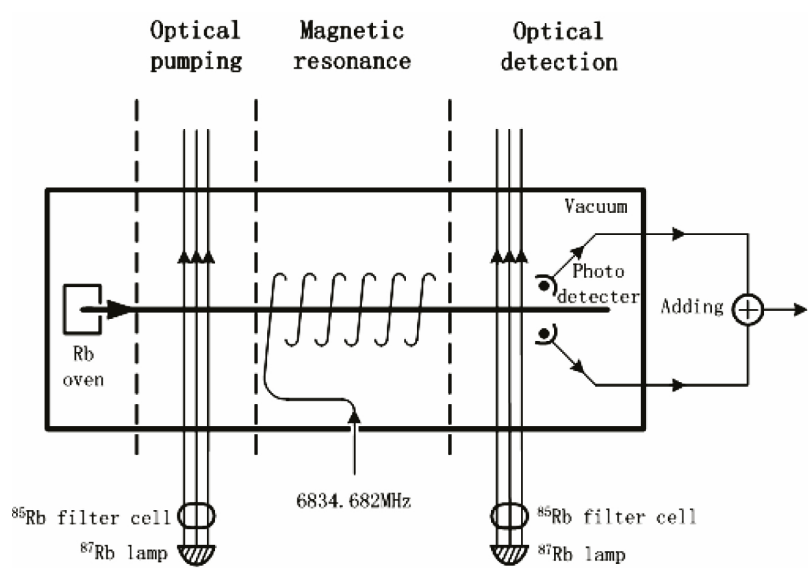

FIG. 2 Structure of lamp-pumping and fluorescence-detection rubidium beam clock.

orescence signal, and the photo-detectors' output can be combined to further improve the $\mathrm{S} / \mathrm{N}$. The signal is proportional to the numbers of detectors used, but the noise is proportional to the square root of the detector numbers. Therefore, more detectors also can improve the $\mathrm{S} / \mathrm{N}$. Taking into account the actual available vacuum space, we estimate that a reasonable number for the detectors is around 10, which means the S/N is improved by a factor of 3 .

\section{OPERATION AS AN ATOMIC CLOCK}

The operation of the rubidium beam clock includes not only optically pumping and optical detection, but also microwave interrogation. For optical pumping, ${ }^{87} \mathrm{Rb}$ lamp produces a and $\mathrm{b}$ lines. After passing through an ${ }^{85} \mathrm{Rb}$ enriched atomic filter, only $\mathrm{b}$ light is retained and will pump ${ }^{87} \mathrm{Rb}$ atoms in the beam to $\mathrm{F}=2$ state. In the microwave interrogation region, for a traditional beam clock, separate microwave interaction regions are used to get Ramsey fringes and to suppress the spectrum linewidth. Here, we propose to use the microwave slow-wave structure in order to make the whole system more compact as shown in Figure 2. The microwave slow-wave structure is a widely used helical type one $[9,10]$, where the microwave field propagates along the wire of the helix. The other end of the helix is grounded. Therefore, the microwave is completely reflected and a standing wave field forms inside the structure. For the Ramsey type beam clock the linewidth is inversely proportional to the distance between the two microwave interaction regions. For the microwave slow-wave structure the linewidth depends on the total effective length of the wire. This design has been widely used in traditional rubidium gas cell clocks, and is much more compact. For the optical detection region the same optical lamp produces the $b$ light to detect the population of $\mathrm{F}=1$ state of ${ }^{87} \mathrm{Rb}$ atoms. Multiple photo-detectors are placed around the detection region. The maximum of fluorescence occurs when the microwave signal is at the resonance frequency of the hyperfine transition. This in turn can be used to feedback control the output frequency of a low phase noise quartz oscillator. The output of the quartz oscillator constitutes the atomic clock output signal.

\section{DISCUSSION}

In this section we discuss and compare the proposed rubidium beam clock scheme with other types of beam clocks. First, why do we choose rubidium and not cesium? The utilization rate of rubidium and magnetic field selection type cesium beam clocks is different. Assuming both of them have same atomic density and pumping efficiency from the ovens, we can only use $1 / 16$ cesium atoms in magnetic field selection type clocks due to cesium 16 ground state sub-levels. If we take into account the velocity selecting device, the final utilization rate is only $3 \%$ for cesium. For rubidium the utilization rate is $5.6 \%$, which is almost two times better in comparison with cesium. In addition, the beam structure is greatly simplified without two sets of magnets. The beam collimation is also simpler, and the required magnet shielding is considerably reduced. This will make the whole beam clock design much lighter.

Second, what are the differences and advantages in comparison with the scheme of Arditi et al. [1]? The main differences can be summarized as following:

- The pumping light is generated by a ${ }^{87} \mathrm{Rb}$ lamp and ${ }^{85} \mathrm{Rb}$ filter cell.

- The diameter of lamps for optical pumping and detection is enlarged to increase the energy density of pumping and detection light.

- Microwave interrogation is performed with a simple long helix structure.

- Fluorescence detection is used instead of transmission detection.

- Multiple photo-detectors are parallel connected to improve the $S / \mathrm{N}$.

Except for the third change, other changes are for the improvement of the $\mathrm{S} / \mathrm{N}$. The total improvement should be more than three orders of magnitude. The theoretical analysis estimates that the short-term stability for the scheme of Arditi et al. [1] is $\sigma(\tau)=10^{-10} / \sqrt{\tau}$ ( $\tau$ is the sampling time). The experimental result is $\sigma(\tau)=2 \times 10^{-9} / \sqrt{\tau}(1 \mathrm{~s}<\tau<100 \mathrm{~s})$. Therefore, it is expected that our scheme should have $\sigma(\tau)=2 \times 10^{-12} / \sqrt{\tau}$. This performance level is already better with traditional magnetic field selected type cesium beam atomic clock but with much compact design due to the third change.

Third, what's the difference between laser pumping and lamp pumping? The advancement of stable, monochromatic, and tunable diode lasers in the near infrared had raised the hope of increasing the $\mathrm{S} / \mathrm{N}$ in optically pumped atomic beam clocks and cell clocks. In theory, laser pumping efficiency could be 4-5 orders of magnitude higher than lamp pumping. This attracted many groups to evaluate the possibilities of laser pumping compared to that of lamp pumping. Unfortunately, the results were not too encouraging. One main problem is due to the frequency fluctuation of the lasers. Currently, only one group in NIST using sophisticated laser technique achieved a relatively good short-term stability performance in 
$30 \mathrm{~s}$ [11]. There are still no further good results for long-term stability performance. In comparison with an optical pumping cesium beam clock, due to a fortuitous coincidence between spectra of ${ }^{87} \mathrm{Rb}$ and ${ }^{85} \mathrm{Rb}$, optical pumping for rubidium beam clocks can be achieved by using a simple discharge lamp. This is not possible with an optical pumping cesium beam clock. A laser will be required to perform the optical pumping. As we have discussed, laser pumping has its own reliability and long-term stability issue. On the other hand, the rubidium discharge lamp technique is more mature and reliable. It is better suited for the development of commercial products in the future.

Fourth, what about the light shift introduced by lamp pumping? Just like laser pumped cesium beam clocks, lamp pumped rubidium beam clocks will cause a light shift when the pumping light leaks into the microwave interrogation region. This can be reduced by a suitable design to block the scattering light. Recently, AIST [4] and NIST [12] proved that the effect of light shift could be reduced to less than $3 \times 10^{-15}$. Consequently, we do not expect light shift to be a serious problem for the performance level we are shooting for.

\section{CONCLUSION}

The proposed scheme of a lamp-pumping fluorescencedetection rubidium beam clock has the advantages of a high pumping efficiency, compact structure, light weight, and good magnet field shielding in comparison with magnetic field selected type cesium beam clocks. It is also more practical than laser pumping beam clocks. From the theoretical analysis, the stability performance level potentially can be three orders of magnitude higher than the former design, and is comparable with commercial cesium beam clocks. We hope this paper can renew interest in the development of compact, portable, and marketable optical pumping rubidium beam clocks.

\section{ACKNOWLEDGEMENTS}

This work is supported by "the Fundamental Research Funds for the Central Universities".

\section{References}

[1] M. Arditi, and P. Cerez, "Hyperfine structure separation of the ground state of ${ }^{87} \mathrm{Rb}$ measured with an optically pumped atomic beam" IEEE T. Instrum. Meas. IM21(4), 391-395 (1972).

[2] P. Cerez, and F. Hartmann, "Theoretical analysis of the operation of an optically pumped Rubidium atomic beam clock" IEEE J. Quantum Elect. QE13(5), 344-351 (1977).

[3] C. Sallot, M. Baldy, D Gin, and R. Petit, " $3 \cdot 10^{-12} \cdot \tau^{-1 / 2}$ on industrial prototype optically pumped Cesium beam frequency standard" in Proceedings of Frequency Control Symposium and PDA Exhibition, 100-104 (IEEE, Tampa, 2003).

[4] K. Hagimoto, Y. Koga, and T. Ikegami, "Reevaluation of the optically pumped Cesium frequency standard NRLM- 4 with an H-bend ring cavity" IEEE T. Instrum. Meas. 57(10), 2212-2217 (2008).
[5] I. Pascaru, "Atomic frequency standard using optical pumping for state preparation and magnetic state selection of atoms" United States Patent 5107226 (1992).

[6] S. Lecomte, M. Haldimann, R. Ruffieux, P. Berthoud, and P. Thomann, "Performance demonstration of a compact, single optical frequency Cesium beam clock for space applications" in Proceedings of Frequency Control Symposium, 1127-1131 (IEEE, Ceneva, 2007).

[7] A. Besedina, A. Gevorkyan, G. Mileti, V. Zholnerov, and A. Bassevich, "Preliminary results of investigation of the high-stable Rubidium atomic beam frequency standard with laser pumping / detection for space application" in Proceedings of 2oth European Frequency and Time Forum, 270-276 (EFTF, Braunschweig, 2006).

[8] T. S. West, and X. K. Williams, "Atomic fluorescence spectroscopy of Silver using a high-intensity hollow cathode lamp as source" Anal. Chem. 40(2), 336-339 (1968).

[9] W. Cai, F. Li, C. Li, and Y. Wang, "Microwave spectra of an atomic beam in a slow-wave structure" Opt. Commun. 161, 227-231 (1999).

[10] J. Yang, Y. Zhang, X. Li, and L. Li, "An improved helical resonator design for Rubidium atomic frequency standards" IEEE T. Instrum. Meas. 59, 1678-1685 (2010).

[11] G. Mileti, J. Q. Deng, F. L. Walls, D. A. Jennings, and R. E. Drullinger, "Laser-pumped Rubidium frequency standards: New analysis and progress" IEEE J. Quantum. Elect. 34(2), 233-237 (1998).

[12] J. H. Shirley, W. D. Lee, and R. E. Drullinger, "Accuracy evaluation of the primary frequency standard NIST-7" Metrologia 38, 427-458 (2001). 